\title{
Erratum to: Reliability and validity of a global question on self-reported chronic morbidity
}

\author{
Johan Van der Heyden • Dirk De Bacquer • \\ Jean Tafforeau $\cdot$ Koen Van Herck
}

Published online: 30 August 2014

(C) Springer-Verlag Berlin Heidelberg 2014

Erratum to: J Public Health (2014) 22:371-380

DOI 10.1007/s10389-014-0624-9

\section{Results}

The original version of this article unfortunately contained a mistake. The prevalences reported by GHQ-score category in Table 1 were swabbed. The corrected Table 1 is given below.

The online version of the original article can be found at $10.1007 /$ s10389-014-0624-9.

J. Van der Heyden $(\bowtie) \cdot J$. Tafforeau

Scientific Institute of Public Health, Unit Surveys, Lifestyle and

Chronic Diseases, 14, Juliette Wytsmanstraat, 1050 Brussels,

Belgium

e-mail: johan.vanderheyden@wiv-isp.be

J. Van der Heyden · D. De Bacquer • K. Van Herck

Department of Public Health, Ghent University, De Pintelaan 185,

9000 Ghent, Belgium 
Table 1 Estimates ${ }^{1}$ and reliability of a general question on self-reported chronic morbidity in HIS and census, population $>14$ years, Belgium, 2001

\begin{tabular}{|c|c|c|c|c|c|c|c|}
\hline \multirow{3}{*}{ Gender } & \multirow[t]{3}{*}{$\mathrm{N}$} & \multicolumn{2}{|c|}{$\begin{array}{l}\text { Prevalence self-reported chronic } \\
\text { morbidity in HIS }\end{array}$} & \multicolumn{2}{|c|}{$\begin{array}{l}\text { Prevalence self-reported chronic } \\
\text { morbidity in census }{ }^{2}\end{array}$} & \multicolumn{2}{|c|}{ Reliability } \\
\hline & & \multirow[t]{2}{*}{$\%$} & \multirow[t]{2}{*}{$(95 \% \mathrm{CI})$} & \multirow[t]{2}{*}{$\%$} & \multirow[t]{2}{*}{$(95 \% \mathrm{CI})$} & \multicolumn{2}{|c|}{ Kappa $(95 \%$ CI) } \\
\hline & & & & & & & \\
\hline Men & 1,408 & 28.3 & $(25.2-31.6)$ & 25.4 & $(22.0-29.2)$ & 0.562 & $(0.527-0.598)$ \\
\hline Women & 1,463 & 28.4 & $(25.1-32.1)$ & 27.2 & $(23.8-30.8)$ & 0.555 & $(0.520-0.590)$ \\
\hline \multicolumn{8}{|l|}{ Age } \\
\hline $15-24$ yrs & 358 & 12.7 & $(8.6-18.5)$ & 10.1 & $(6.3-15.8)$ & 0.456 & $(0.418-0.494)$ \\
\hline $25-44 \mathrm{yrs}$ & 1,083 & 18.2 & $(15.2-21.8)$ & 13.1 & $(10.5-16.4)$ & 0.506 & $(0.469-0.542)$ \\
\hline $45-64 \mathrm{yrs}$ & 839 & 32.7 & $(28.4-37.3)$ & 31.4 & $(26.9-36.3)$ & 0.540 & $(0.504-0.576)$ \\
\hline $65+\mathrm{yrs}$ & 591 & 50.4 & $(43.9-56.9)$ & 54.3 & $(47.1-61.4)$ & 0.474 & $(0.437-0.511)$ \\
\hline \multicolumn{8}{|l|}{ Self-rated health } \\
\hline Very good to good & 1,930 & 15.7 & $(13.3-18.5)$ & 11.8 & $(9.5-14.5)$ & 0.367 & $(0.329-0.407)$ \\
\hline Moderate to bad & 658 & 68.0 & $(63.0-72.5)$ & 68.4 & $(63.5-73.0)$ & 0.427 & $(0.389-0.465)$ \\
\hline \multicolumn{8}{|l|}{ GHQ-score } \\
\hline $0-3$ & 2,197 & 24.7 & $(22.1-27.6)$ & 22.5 & $(19.8-25.5)$ & 0.526 & $(0.488-0.565)$ \\
\hline 4 or more & 341 & 47.6 & $(40.1-55.1)$ & 42.1 & $(34.6-49.9)$ & 0.631 & $(0.596-0.667)$ \\
\hline \multicolumn{8}{|l|}{ Education } \\
\hline Primary & 475 & 39.4 & $(32.4-46.9)$ & 49.4 & $(40.3-58.6)$ & 0.563 & $(0.527-0.599)$ \\
\hline Lower secondary & 569 & 36.2 & $(30.3-42.5)$ & 32.8 & $(26.8-39.3)$ & 0.506 & $(0.469-0.544)$ \\
\hline Higher secondary & 784 & 23.7 & $(19.4-28.6)$ & 19.1 & $(15.4-23.5)$ & 0.590 & $(0.555-0.625)$ \\
\hline Tertiary & 929 & 21.3 & $(17.9-25.0)$ & 16.2 & $(13.1-19.9)$ & 0.523 & $(0.486-0.560)$ \\
\hline \multicolumn{8}{|l|}{ Nationality } \\
\hline Belgian & 2,591 & 28.7 & $(26.1-31.4)$ & 26.7 & (23.9-29.8) & 0.574 & $(0.539-0.609)$ \\
\hline Non-Belgian & 277 & 23.4 & $(17.3-30.9)$ & 20.3 & $(14.4-27.8)$ & 0.417 & $(0.379-0.456)$ \\
\hline \multicolumn{8}{|l|}{ HIS respondent } \\
\hline Selected person & 2,755 & 28.2 & $(25.7-30.9)$ & 25.8 & $(23.2-28.5)$ & 0.559 & $(0.523-0.594)$ \\
\hline Proxy & 112 & 32.3 & $(19.1-49.2)$ & 39.2 & $(23.7-57.2)$ & 0.555 & $(0.519-0.590)$ \\
\hline Total & 2,871 & 28.4 & $(25.9-30.9)$ & 26.3 & $(23.6-29.2)$ & 0.559 & $(0.523-0.594)$ \\
\hline
\end{tabular}

1 weighted percentages

2 calculated among HIS respondents only 